\title{
PORIFERA (DEMOSPONGIAE, CALCAREA) ENTRE OS ESTADOS DO CEARÁ E PERNAMBUCO, BRASIL . TAXONOMIA E DISTRIBUIÇÃO. ${ }^{1}$
}

\author{
Josivete Pinheiro dos SANTOS ${ }^{2}$ \\ Beatriz MOTHES ${ }^{3}$ \\ Deusinete de Oliveira TENÓRIO ${ }^{2}$ \\ Jerrana CANTARELLI ${ }^{2}$ \\ Trabalho realizado com o auxílio do $\mathrm{CNPq}^{1}$ \\ Departamento de Oceanografia da UFPE ${ }^{2}$ \\ Fundação Zoobotânica do Rio Grande do Sul ${ }^{3}$ \\ (e-mail: josivete@ zaz.com.br)
}

\section{RESUMO}

Estudos sobre poríferos da costa Nordeste do Brasil foram realizados dentro do programa REVIZEE NE- 1, em expedição feita pelo Navio Oceanográfico Antares, durante a pernada 6 , entre as coordenadas geográficas de $3^{\circ} 54^{\prime}$ e $7^{\circ} 48^{\prime} \mathrm{S}$ e $34^{\circ} 34^{\prime}$ e $37^{\circ} 38^{\prime} \mathrm{W}$, no período de 12 a 16 de outubro de 1995. As amostras foram adquiridas através de dragagens, em 8 estações de coleta, em profundidades entre 42 e 166 metros; fixadas no local com formol neutro a $4 \%$ e em laboratório conservadas em álcool a 98\%. Dados hidrológicos foram obtidos simultaneamente. A salinidade variou de $36,18 \%$ o a $37,10 \%$ o e a temperatura de $23,54^{\circ} \mathrm{C}$ a $26,48^{\circ} \mathrm{C}$. A taxonomia das espécies baseou-se no estudo das escleras e/ou fibras através da dissociação espicular, cortes histológicos e observação do conjunto espicular no microscópio eletrônico de varredura. Os espécimes encontram-se depositados na Coleção de Bentos do Departamento de Oceanografia da Universidade Federal de Pernambuco e duplicados na Coleção de Poríferos Marinhos do Museu de Ciências Naturais da Fundação Zoobotânica do Rio Grande do Sul. Registrou-se a presença de Calcarea e Demospongiae. Os táxons identificados de Demospongiae são: HOMOSCLEROPHORIDA (Plakinidae) Plakinastrella Schulze,1880 e Plakortis Schulze,1880; SPIROPHORIDA (Tetillidae) Cinachyrella alloclada (Uliczka,1929); ASTROPHORIDA (Geodiidae) Erylus formosus Sollas,1886, Geodia gibberosa Lamarck,1815 e (Ancorinidae) Stelletta Schmidt,1862; HADROMERIDA (Spirastrellidae) Spirastrella Schmidt,1868; AGELASIDA (Agelasidae) Agelas dispar Duchassaing \& Michelotti,1864; HALICHONDRIDA (Halichondriidae) Halichondria Fleming,1828; HAPLOSCLERIDA (Niphatidae) Niphates Duchassaing \& Michelotti,1864 e (Chalinidae) Haliclona Grant,1835; DICTYOCERATIDA (Spongiidae) Hyattella Lendenfeld,1888 e (Ircinidae) Ircinia Nardo,1833; VERONGIDA (Aplysinidae) Aplysina fistularis fulva (Pallas,1766). As espécies apresentaram alta diversidade nas estações 210 e 215. A associação das amostras apresentou dois grupos, sendo a diversidade de espécies o fator a separá-las. O primeiro caracterizado pela maior diversidade e o segundo pela baixa diversidade. A associação das espécies apresentou quatro grupos distintos, variando de acordo com a profundidade.

Palavras Chave: Porifera, Demospongiae, Calcarea, Plataforma Continental Nordeste, Brasil . 


\section{ABSTRACT \\ Porifera (Demospongiae, Calcarea) between Ceara and Pernambuco States, Brazil. Taxonomy and Distribution.}

Porifera of the Northeastern Brazil was studied as part of the Cooperation Research Program "Live Resources of the Economic Exclusive Zone - Northeastern I " (REVIZEE NE-I) - leg 6. Samples were collected by the Oceanographic Ship Antares, from October 12 to 16,1995 , at $3^{\circ} 54^{\prime}-7^{\circ} 48^{\prime} \mathrm{S}$ and $34^{\circ} 34^{\prime}-37^{\circ} 38^{\prime} \mathrm{W}$, in depths between 42 and 166 meters. Fixation was made with neutralized 4\% formaldehyde and alcohool. Concurrent hydrological data were obtained. Salinity varied from $36,18 \%$ o to $37,10 \%$, and the temperature from $23,54^{\circ} \mathrm{C}$ to $26,48^{\circ} \mathrm{C}$. The taxonomic study was accomplished with the study of the spicules and fibers through the dissociation by eletronic microscopy. The studied specimens are deposited in the Benthos Collection of the Department of Oceanografy of the Federal University of Pernambuco and duplicated in the Collection of Marine Porifera of the Natural Sciences Museum of the Fundação Zoobotânica of Rio Grande do Sul. It was identified: Demospongiae : HOMOSCLEROPHORIDA (Plakinidae) Plakinastrella Schulze, 1880 e Plakortis Schulze,1880; SPIROPHORIDA (Tetillidae) Cinachyrella alloclada (Uliczka,1929); ASTROPHORIDA (Geodiidae) Erylus formosus Sollas,1886, Geodia gibberosa Lamarck,1815 e (Ancorinidae) Stelletta Schmidt,1862; HADROMERIDA (Spirastrellidae) Spirastrella Schmidt,1868; AGELASIDA (Agelasidae) Agelas dispar Duchassaing \& Michelotti,1864; HALICHONDRIDA (Halichondriidae) Halichondria Fleming,1828; HAPLOSCLERIDA (Niphatidae) Niphates Duchassaing \& Michelotti,1864 e (Chalinidae) Haliclona Grant,1835; DICTYOCERATIDA (Spongiidae) Hyatella Lendenfeld,1888 e (Ircinidae) Ircinia Nardo,1833; VERONGIDA (Aplysinidae) Aplysina fistularis fulva (Pallas,1766) and Calcarea. Highest diversity was registered ta station 210 and 215. Samples association presented two groups: the first characterized by the higher diversity and the second by the low diversity. The association of the species presented four groups, varying according to the depth.

Key Words : Porifera, Demospongiae, Calcarea, Northeastern Continental Shelf, Brazil.

\section{INTRODUÇÃO}

Poríferos são animais multicelulares, filtradores por excelência e detentores de células denominadas coanócitos. Exclusivamente aquáticos, a maior parte é marinha. Os que habitam o bentos marinho tem sua distribuição espacial compreendida desde o infralitoral até a região hadal, da linha do equador aos pólos. Durante grande parte de suas vidas constituem formas sésseis, e apenas num curto período de sua existência são livres natantes, apresentando uma larva pelágica com duração variável de algumas horas. Vivem fixos em substratos sólidos tais como rochas, conchas de moluscos, tubos de poliquetos, em raízes de mangues, em recifes de corais e em algas calcáreas, e 
também em fundos móveis tais como lodo e areia fina, onde espécies que apresentam estruturas espiculares em formato de âncora fixam-se. Apresentam maior diversidade em águas tropicais (Bergquist, 1978).

Demospongiae é a classe mais representativa dentre os poríferos. Várias famílias merecem destaque, como as perfuradoras Clionidae, que podem perfurar estruturas calcáreas como corais ou conchas de moluscos para depois se fixar nos canais perfuradores. Essas esponjas apresentam grande importância ecológica, pois transformam as rochas calcáreas em cascalho ou areia, ou seja, em fundos biodetríticos (Bergquist, 1978).

Algumas esponjas, particularmente aquelas que crescem em recifes de coral, apresentam simbiose com cianobactérias. Esta simbiose aumenta imensamente a taxa de crescimento da esponja e habilidade de competição nos recifes de coral (Hooper,1998).

Algumas espécies de esponjas apresentam ação antimicrobiana, como Verongia aerophoba, Aplysilla sulfurea, Dysidea herbacea e Phakellia flabellata, que são capazes de inibir bactérias gram + e gram -. Burkholder (1969) afirmou que Verongia aerophoba é capaz de inibir a bactérias Escherichia coli e o fungo Candida albicans.

Estudos com atividade biológica de poríferos da costa brasileira iniciaram, conforme Kelecon (1991) com Raymond Zelnik no Instituto Butantã, que estudou algumas espécies em Abrolhos (BA), Guaraparí (ES), Arraial do Cabo (RJ), Itaipu (RJ) e Itacuruça (RJ), investigando a presença de esteróis e metabólitos de bromotirosina. Kelecon (1991) afirmou que a espécie Xestospongia sapra apresenta ação vasodilatadora devido aos alcalóides liberados em seu extrato, Mycale sp. uma forte ação antiviral e Dsysidea avara ação citotóxica para drogas anti-HIV. Muricy et al (1993) testaram extratos de 21 espécies de esponjas e constataram que 19 inibiram o crescimento de microorganismos em até $50 \%$, mostrando forte atividade antibacteriana e antifúngica. Berlink et al (1995) analisaram os extratos hidroalcoólicos de Haliclona erina e concluiram que esta apresenta atividade citotóxica e hemolítica. Hooper (1998) afirmou que a esponja Tedania charcoti produz um antibiótico extremamente efetivo, e as espécies Cynbastela hooper e Cynbastela colalliophila apresentam um composto anti-malarial de potencial considerável para uso farmacêutico. Mais recentemente, um estudo pioneiro no país está sendo desenvolvido entre pesquisadores da Fundação Zoobotânica do Rio Grande do Sul, Universidade Federal do Rio Grande do Sul, Hospital das Clínicas de Porto Alegre e a SOAD: Fundação Central Sul-americana para o Desenvolvimento de Novas Drogas Anticâncer-National Cancer Institute, EUA; objetivando a descoberta de novas drogas anti-câncer e anti-HIV. Os primeiros resultados encontram-se em Lerner et al. (1998 e 1999).

Também atividade anti-incrustante vêm sendo pesquisada em esponjas. Conforme Gama \& Pereira (1996) substâncias sintetizadas por elas são potenciais ativos para a fabricação de novas tintas, capazes de controlar a incrustação sem danificar o meio ambiente.

Os poríferos brasileiros são ainda pouco conhecidos, principalmente os de águas profundas. O programa "Recursos Vivos da Zona Econômica Exclusiva" REVIZEE - que tem como um dos objetivos a identificação de espécies que habitam a plataforma continental e o fundo marinho do talude superior, serviu de base para 
elaboração deste trabalho, no qual está inserido a Universidade Federal de Pernambuco, através do Departamento de Oceanografia.

Esta pesquisa pretende contribuir para o conhecimento da taxonomia e distribuição dos Porifera da Zona Econômica Exclusiva do Nordeste Brasileiro.

\section{MATERIAL E MÉTODOS}

As amostras estudadas integram o acervo da coleção de Bentos do Departamento de Oceanografia da Universidade Federal de Pernambuco. Este material foi coletado em 8 estações oceanográficas, pelo Navio Oceanográfico Antares, dentro do Programa de Avaliação do Potencial Sustentável dos Recursos Vivos da Zona Econômica Exclusiva ( REVIZEE NE-I), no período de 12 a 16/10/95, entre os estados do Ceará e Pernambuco (3 $3^{\circ}$ 54,5172' a $7^{\circ} 48,1646^{\prime}$ e $34^{\circ} 29,2315^{\prime}$ a $37^{\circ}$ 38,0082') (Figura 1). Os exemplares foram recolhidos através de draga retangular, com um tempo de arrasto que variou de 2 a 5 minutos e velocidade de 2 nós, em profundidades que variaram entre 42 e 166 metros. Simultaneamente, foram obtidos dados de salinidade e temperatura da água. Os exemplares foram fixados com formol neutro a 4\% no local, e em laboratório com álcool absoluto (98\%).

Em laboratório, foi realizado o estudo taxonômico das demospongias com base na observação das escleras e/ou fibras, com as técnicas de dissociação espicular, elaboração de lâminas histológicas e cortes diafanizados, de acordo com Mothes (1996). As lâminas foram observadas com o auxílio de um microscópio óptico Leitz Laborlux K, onde efetuou-se mensurações micrométricas do conjunto espicular de cada espécime, com aplicação da "Fórmula de Sturges", toda vez que fora detectada mais de uma classe espicular. Escleras inteiras, bem como suas extremidades e da arquitetura esqueletal foram analisadas e fotografadas em Microscopia Eletrônica de Varredura Jeol JSM 5200. A identificação das esponjas calcárias foi fundamentada na observação das escleras, através da preparação espicular utilizando-se hipoclorito de sódio (Solange Peixinho e Silva, comunicação pessoal)

A classificação taxonômica segue Hooper (1998).

A análise multivariada baseou-se na presença/ausência das espécies, sendo utilizado o índice de Jaccard (1908); o método de ligação foi o do peso proporcional WPGMA (Weight Pair Group Mathematical-Arithmetic Average) (Rohlf \& Fisher, 1968). Foi realizada análise cofenética para verificar significância dos dendrogramas.

As esponjas de natureza calcária foram até o momento identificadas somente até o nível de classe.

\section{RESULTADOS}

Foram identificadas: 2 classes, 3 subclasses, 9 ordens, 12 famílias, 14 gêneros e 5 espécies, abaixo discriminadas:

\section{Classe Calcarea \\ Classe Demospongiae}

Subclasse Homoscleromorpha Lévi, 1973 
Ordem Homosclerophorida Dendy , 1905

Família Plakinidae Schulze , 1880

Plakortis Schulze, 1880

Plakinastrella Schulze, 1880

Subclasse Tetractinomorpha Lévi, 1973

Ordem Spirophorida Lévi, 1973

Família Tetillidae Sollas, 1886

Cinachyrella alloclada (Uliczka, 1929)

Ordem Astrophorida Lévi, 1973

Família Geodiidae Gray, 1867

Erylus formosus Sollas, 1886

Geodia gibberosa Lamarck, 1815

Família Ancorinidae Schmidt, 1870

Stelletta Schmidt, 1862

Ordem Hadromerida Topsent, 1898

Família Spirastrellidae Ridley \& Dendy, 1886.

Spirastrella Schmidt, 1868.

Subclasse Ceractinomorpha Lévi, 1973

Ordem Agelasida Hartman, 1982

Família Agelasidae Verril, 1907

Agelas dispar Duchassaing \& Michelotti, 1864.

Ordem Halichondrida Vosmaer, 1885

Família Halichondriidae Vosmaer, 1887

Halichondria Fleming, 1828

Ordem Haplosclerida Topsent, 1928

Família Chalinidae Gray, 1867

Haliclona Grant , 1835

Família Niphatidae Van Soest, 1980

Niphates Duchassaing \& Michelotti, 1864

Ordem Dictyoceratida Minchin, 1900

Família Spongiidae Gray, 1867

Hyattella Lendenfeld, 1888

Família Ircinidae Gray, 1867

Ircinia Nardo, 1833

Ordem Verongida Bergquist, 1980

Família Aplysinidae Carter, 1875.

Aplysina fistularis fulva (Pallas, 1766)

A temperatura da água apresentou uma amplitude de $2,94^{\circ} \mathrm{C}$, com valor máximo de $26,48^{\circ} \mathrm{C}$ (Estação 218 - RN) e o valor mínimo de $23,54^{\circ} \mathrm{C}$ (Estação 220 $\mathrm{RN}$ ) ; e a salinidade variou entre 36,18\%o (Estação 208 - CE) e 37,10\%o (Estação 225 - RN) com amplitude menor que 1,00\%o (Tabela 1) Indicando encontrar-se em área onde atua a Água Tropical.

A profundidade variou de 42 a 166 metros, tendo todas as amostras sido coletadas na Plataforma e Talude Continental. 
As espécies que apresentaram maior frequência de ocorrência foram Halichondria sp. (62,5\%), Agelas dispar Duchassaing \& Michelotti,1864 (50,0\%) e Aplysina fistularis fulva (Pallas,1766) (50,0\%). A diversidade das espécies mostrou-se muito baixa nas estações 218,220 e 235 (< 1,0 bits. ind $\left.{ }^{-1}\right)$, baixa nas estações 208 e $225\left(1,0-2,0\right.$ bits. ind $\left.^{-1}\right)$, média na estação $213\left(2,0-3,0\right.$ bits. ind $\left.^{-1}\right)$ e alta nas estações 210 e 215 ( >3,0 bits. ind ${ }^{-1}$ ) (Tabela 2). Destaca-se contudo, que o pequeno número de amostras só permite referir-se ao registro, podendo numa malha maior de amostragem serem encontradas maiores ocorrência de outras espécies.

Os espécimes da classe Calcarea foram registrados nos estados do Ceará (Estação 210), Rio Grande do Norte (Estações 215 e 218) e Pernambuco (Estação 235), entre profundidades de 42 e 166 metros.

A análise cofenética da associação das estações mostrou que há diferenças significativas entre as mesmas com $\mathrm{r}=0,95$, evidenciando dois grupos: o primeiro agrupando as estações 210 e 215, com maior diversidade de espécies e associado às maiores profundidades (166 metros); e o segundo agrupando as estações 218 e 235, com diversidade baixa e menores profundidades $(<50$ metros ) (Figura 2$)$.

A análise cofenética da associação das espécies apresentou um $r=0,77$, portanto significativa. Evidenciaram-se quatro grupos: o primeiro foi representado pelas espécies Plakortis sp., Agelas dispar Duchassaing \& Michelotti,1864, Halichondria sp., Plakinastrella sp. e Aplysina fistularis fulva (Pallas,1766); registradas em profundidades entre 50 e 166 metros, consideradas euribatas; o segundo pelas espécies Stelletta sp., Niphates sp., Geodia gibberosa Lamarck,1815 e Ircinia sp. que foram registradas em profundidades entre 70 e 166 metros, classificadas como profundas; o terceiro pelas espécies Spirastrella sp. e Haliclona sp., registradas em profundidades em torno de 166 metros, consideradas profundas; e o quarto grupo pelas espécies Cinachyrella alloclada (Uliczka,1929) e Erylus formosus Sollas,1886, presentes em profundidades entre 40 e 55 metros, sendo consideradas rasas (Figura 3).

\section{COMENTÁRIOS E CONCLUSÕES}

Amplia-se no presente estudo o registro de Demospongiae para a costa brasileira (Tabela 3). Ao largo da costa do Rio Grande do Norte esta fauna bentônica é pela primeira vez estudada. Para a costa do Ceará os únicos estudos até o presente encontram-se em: Laubenfels (1956) registra algumas espécies após o estudo realizado em espécimes conservados em seco, coletados entre 5 a 7 metros de profundidade, próximo à Camocim; Johnson (1971) identifica alguns táxons ao largo da costa e outros para a praia de Mucuripe, sem determinação de profundidade; e Hechtel (1976) ao estudar espécimes da coleção de Foster-Laborel cita a ocorrência de Agelas sparsus (Gray, 1867) para Fortaleza, sem anotação de profundidade. Para a região supra citada, registra-se pela primeira vez as espécies Erylus formosus Sollas, 1886 e Agelas dispar Duchassaing \& Michelotti,1864 como também os gêneros Plakinastrella Schulze,1889, Spirastrella Schmidt,1868, Niphates Duchassaing \& Michelotti,1864 e Hyattella Lendelfeld,1888. Confirma-se a presença de Aplysina fistularis fulva (Pallas, 1766) citada como Verongia fulva por Johnson (1971), além dos gêneros Halichondria Fleming,1828, Haliclona Grant,1835 e Ircinia Nardo,1833. 
Ao largo da costa do Ceará registra-se pela primeira vez Demospongiae em águas $>100 \mathrm{~m}$ de profundidade e neste estoque estão Plakinastrella sp., Spirastrella sp., Halichondria sp., Niphates sp., Haliclona sp., Hyattella sp. e Ircinia sp., e ao mesmo tempo amplia-se o registro da distribuição batimétrica de Aplysina fistularis fulva (Pallas, 1766) e Agelas dispar Duchassaing \& Michelotti, 1864.

A distribuição das espécies de esponjas é determinada principalmente pelo tipo de substrato, entrada de sedimentos, temperatura e ação das ondas (Sarà \& Vacelet, 1973). Todas as espécies do presente estudo foram provenientes de sedimento biodetrítico e cascalho, tendo a profundidade maior influência.

Entre as espécies identificadas apenas Halichondria sp. Agelas dispar Duchassaing \& Michelotti,1864 e Aplysina fistularis fulva (Pallas,1766) apresentaram alta frequência de ocorrência .As estações 210 e 215 possuiram uma maior diversidade de espécies, indicando aumento das espécies com a profundidade.

A análise cofenética de associação das estações mostrou que há diferenças significativa, formando-se dois grupos. O primeiro mostrando as estações de maior diversidade ( 210 e 215 ), e o segundo as estações de menor diversidade (218 e 235).

A análise cofenética de associação das espécies revelou a formação de quatro grupos distintos, variando de acordo com a profundidade.

Tabela 1 - Parâmetros ambientais das amostras de porifera do revizee ne-1 entre os estados do Ceará e Pernambuco - Brasil.

\begin{tabular}{ccccccc}
\hline \hline ESTAÇÃOO & DATA & LAT. (S) & LONG. (W) PROF. (m) & $\begin{array}{c}\text { TEMP. } \\
\left({ }^{\circ} \mathbf{C}\right)\end{array}$ & SAL. (\%o) \\
\hline 208 & $12 / 10 / 95$ & $3^{\circ} 54,5172^{\prime}$ & $37^{\circ} 38,0082^{\prime}$ & 43,6 & 26,34 & 36,18 \\
210 & $12 / 10 / 95$ & $3^{\circ} 50,5598^{\prime}$ & $37^{\circ} 37,6930^{\prime}$ & 166 & 25,78 & 36,48 \\
213 & $13 / 10 / 95$ & $4^{\circ} 38,7100^{\prime}$ & $36^{\circ} 44,6629^{\prime}$ & 51,87 & 26,41 & 36,32 \\
215 & $13 / 10 / 95$ & $4^{\circ} 38,4941^{\prime}$ & $36^{\circ} 42,9565^{\prime}$ & 77,49 & 26,30 & 36,25 \\
218 & $13 / 10 / 95$ & $4^{\circ} 47,6951^{\prime}$ & $35^{\circ} 20,6924^{\prime}$ & 44,94 & 26,48 & 36,31 \\
220 & $14 / 10 / 95$ & $4^{\circ} 44,4914^{\prime}$ & $35^{\circ} 16,8625^{\prime}$ & 66,15 & 23,54 & 36,56 \\
225 & $14 / 10 / 95$ & $6^{\circ} 21,6013^{\prime}$ & $34^{\circ} 46,7914^{\prime}$ & 56,78 & 24,91 & 37,10 \\
235 & $16 / 10 / 95$ & $7^{\circ} 48,1646^{\prime}$ & $34^{\circ} 34,1392^{\prime}$ & 42,35 & 26,43 & 36,29 \\
\hline \hline
\end{tabular}


Tabela 2 - Porifera coletados pelo Programa REVIZEE NE-1 entre os Estados do Ceará e Pernambuco - Brasil.

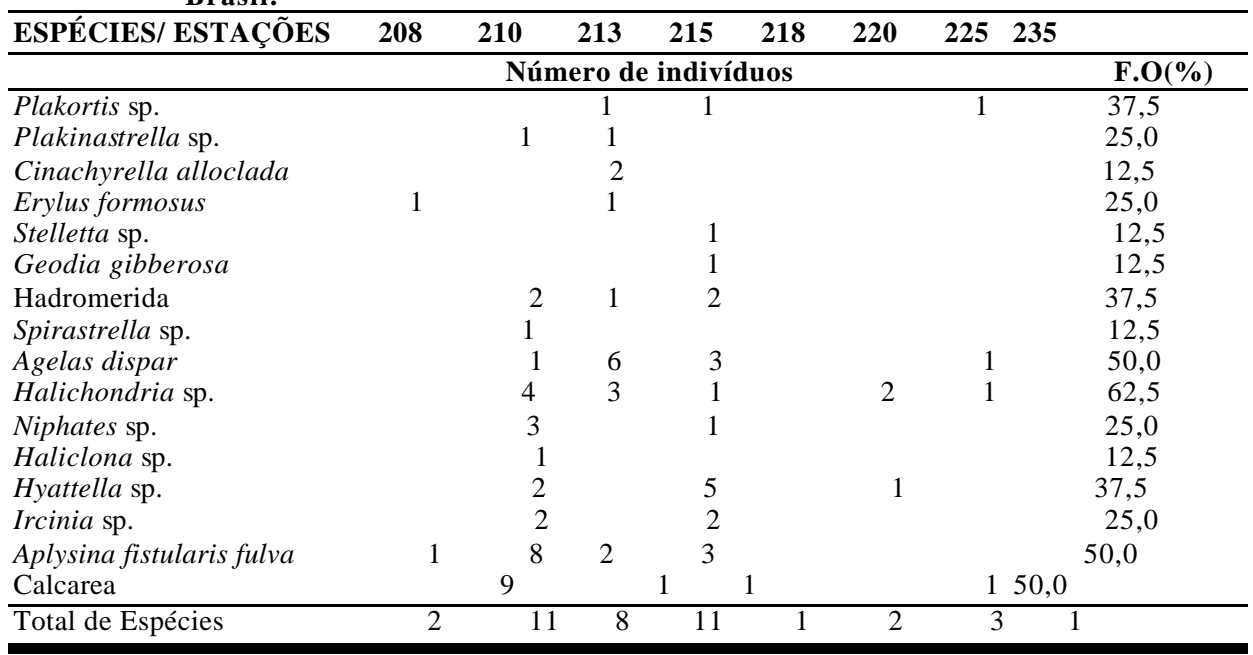


Tabela 3 - Demospongiae registradas na costa do Rio Grande do Norte e Ceará-Brasil. Informações da Literatura.

\begin{tabular}{|c|c|c|c|c|}
\hline $\begin{array}{l}\text { Laubenfels, } \\
1956\end{array}$ & Johnson, 1973 & Hechtel, 1976 & PRESENTE ESTUDO & PRESENTE ESTUDO \\
\hline & & & "Col. REVIZEE-NE-I" & "Col. REVIZEE-NE-I" \\
\hline $\begin{array}{l}\text { "Col."Prof. Dias } \\
\text { da Rocha." }\end{array}$ & $\begin{array}{l}\text { "Col. Laboratório } \\
\text { de Ciências do Mar } \\
\text { UFCE }\end{array}$ & $\begin{array}{l}\text { “Col. Foster-Laborel” } \\
\text { Fortaleza, Ceará }\end{array}$ & Ceará & Rio Grande do Norte \\
\hline Próximo à & (LABOMAR) & & $3^{\circ} 50^{\prime} \mathrm{S}-3^{\circ} 54^{\prime} \mathrm{S}$ e & $4^{\circ} 38^{\prime} \mathrm{S}-6^{\circ} 21^{\prime} \mathrm{S}$ e \\
\hline Camocim, Ceará & & & $37^{\circ} 37^{\prime} \mathrm{W}-37^{\circ} 38^{\prime} \mathrm{W}$ & $34^{\circ} 46^{\prime} \mathrm{W}-37^{\circ} 38^{\prime} \mathrm{W}$ \\
\hline $\begin{array}{l}\text { Próximo a } \\
\mathrm{O} 3^{\circ} \mathrm{S}-41^{\circ} \mathrm{W}\end{array}$ & $\begin{array}{l}\text { ao largo da costa de } \\
\text { Fortaleza, Ceará } \\
03^{\circ} 55 \mathrm{~S}-38^{\circ} 31 \mathrm{~W} \text { e } \\
\text { Praia de Mucuripe, } \\
\text { Ceará }\end{array}$ & & & \\
\hline prof. 5 a $7 \mathrm{~m}$ & prof. águas rasas & & prof. $42-166 \mathrm{~m}$ & prof. $44-77 \mathrm{~m}$ \\
\hline $\begin{array}{l}\text { Hippiospongia } \\
\text { lachne } \\
\text { Ircinia } \\
\text { fascicularis } \\
\text { Halme sp. } \\
\text { Verongia } \\
\text { fistularis } \\
\text { Verongia } \\
\text { longissima } \\
\text { Verongia } \\
\text { capensis } \\
\text { Callyspongia, } \\
\text { "perhaps } \\
\text { Fallax or } \\
\text { Vaginalis" }\end{array}$ & $\begin{array}{l}\text { Polyfibrospongia } \\
\text { sp. } \\
\text { Ircinia fasciculata } \\
\text { Ircinia strobilina } \\
\text { Verongia fulva } \\
\text { Verongia } \\
\text { Longissima } \\
\text { Verongia } \text { sp. } \\
\text { Ianthella iantella } \\
\text { Haliclona } \text { sp. } \\
\text { Callyspongia aff. } \\
\text { Difusa } \\
\text { Fibulia } \text { aff. } \\
\text { Bermuda } \\
\text { Pellina } \text { aff. } \\
\text { Eusiphonia } \text { sp. } \\
\text { Agelas } \text { sp. } \\
\text { Kieplitela } \text { aff. } \\
\text { Antrodes } \text { sp. } \\
\text { Microciona } \\
\text { prolifera } \\
\text { Axinella } \text { sp. } \\
\text { Halichondria } \text { sp. } \\
\text { Hymeniacidon aff. } \\
\text { Dystacta } \text { sp. } \\
\text { Cinachyra } \\
\text { rhizophyta } \\
\text { Cinachyra } \\
\text { alloclada }\end{array}$ & Agelas sparsus & $\begin{array}{l}\text { Plakinastrella } \mathrm{sp} . \\
\text { Erylus formosus } \\
\text { Spirastrella } \mathrm{sp} . \\
\text { Agelas dispar } \\
\text { Halichondria } \mathrm{sp} . \\
\text { Niphates } \mathrm{sp} . \\
\text { Haliclona } \mathrm{sp} . \\
\text { Hyatttella } \text { sp. } \\
\text { Ircinia } \text { sp. } \\
\text { Aplysina fistularis fulva }\end{array}$ & $\begin{array}{l}\text { Plakortis sp. } \\
\text { Plakinastrella } \mathrm{sp} . \\
\text { Cinachyrella alloclada } \\
\text { Erylus formosus } \\
\text { Stelletta } \mathrm{sp} . \\
\text { Geodia gibberosa } \\
\text { Agelas dispar } \\
\text { Halichondria } \mathrm{sp} . \\
\text { Niphates } \mathrm{sp} . \\
\text { Hyattella } \text { sp. } \\
\text { Ircinia } \mathrm{sp} \text {. } \\
\text { Aplysina fistularis fulva }\end{array}$ \\
\hline
\end{tabular}




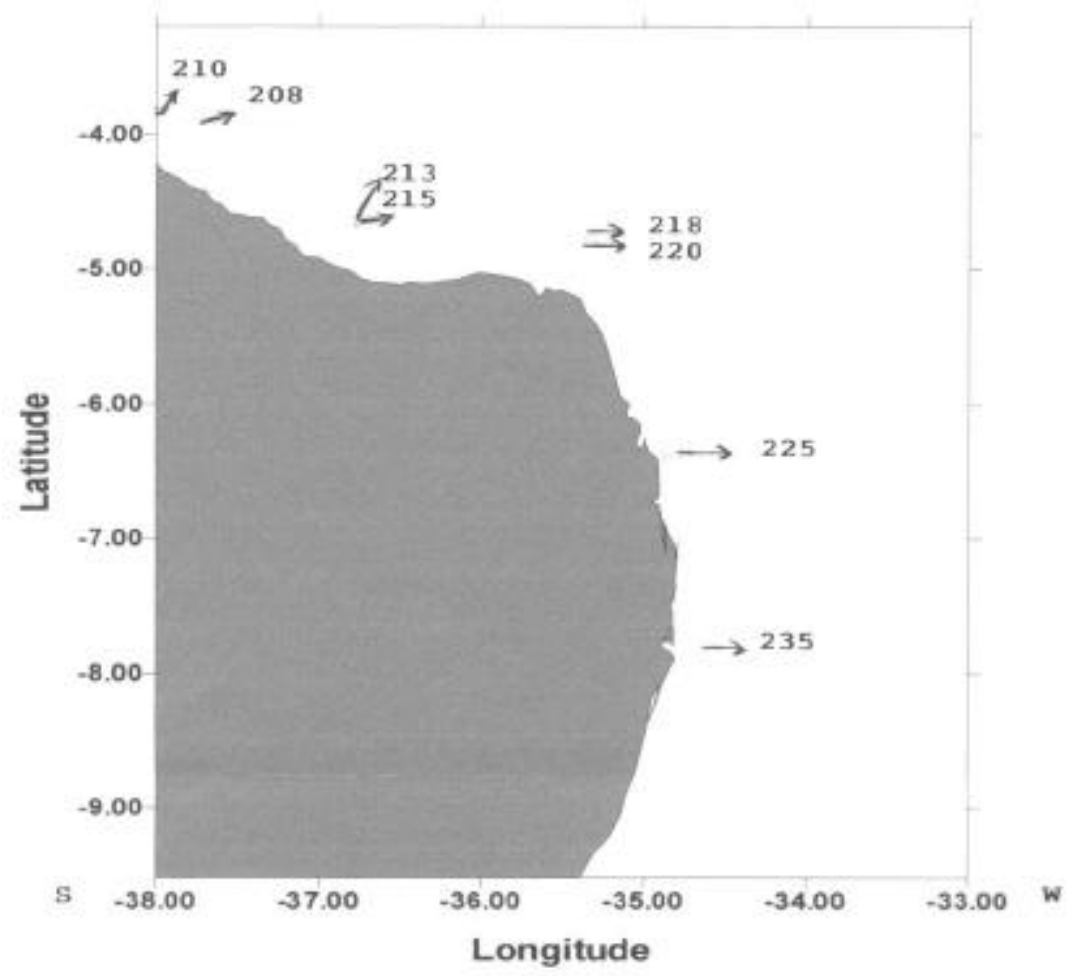

Figura 1- Mapa da área estudada e estações de coleta. 


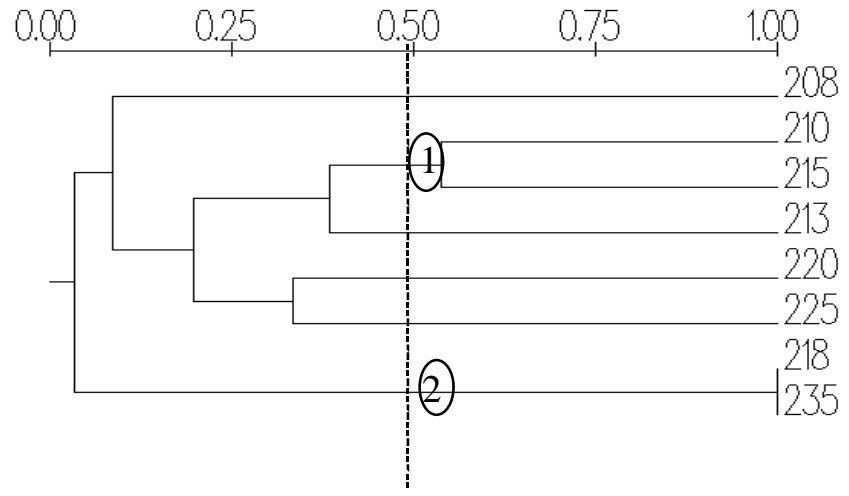

Figura 2 - Dendrograma da associação das amostras do REVIZEE NE -I entre os estados do Ceará e Pernambuco - Brasil.

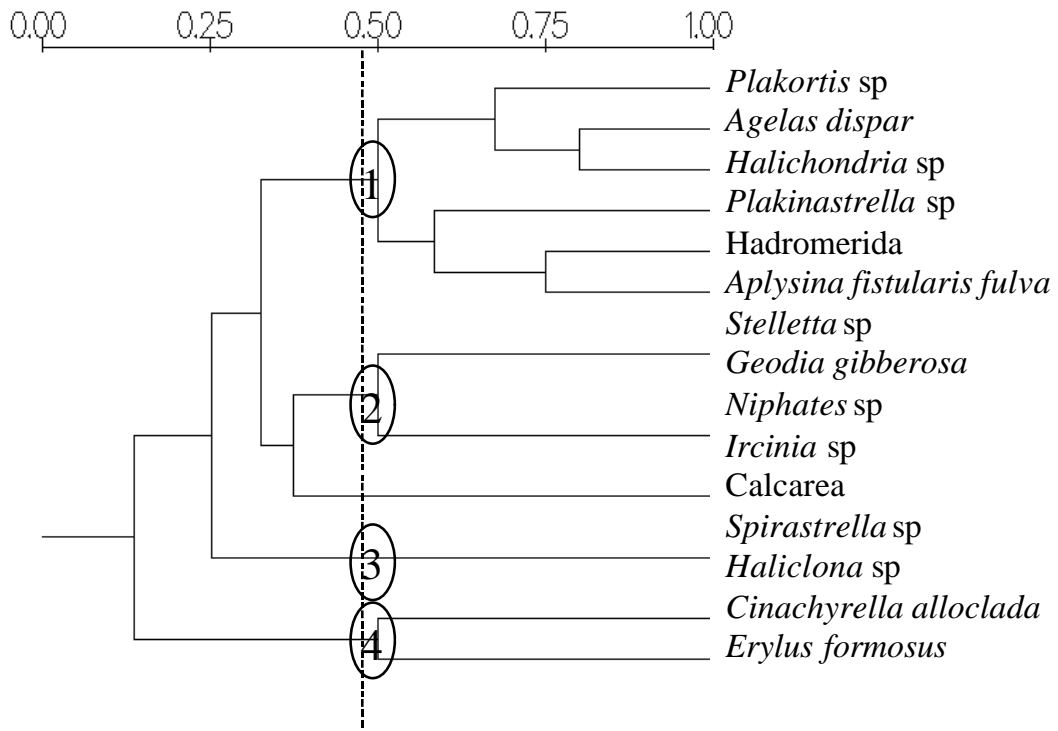

Figura 3 - Dendrograma da associação das espécies de Porifera do REVIZEE NE - I entre os estados do Ceará e Pernambuco - Brasil. 


\section{REFERÊNCIAS BIBLIOGRÁFICAS}

BERLINK , R.G.S.; ALMEIDA, A. M.P. ; OGAWA,C.A; SANCHEZ, M.A.A.; FREITAS, J.C.; MALPEZZI,E.L.A. ; HAJDU, E. Alcalóides Alquilpiridínicos de Esponjas Marinhas. Reunião Anual da Sociedade Brasileira de Química, 18, Caxambu, MG, 1995.

BERGQUIST, P.R Sponges. Los Angeles, University of California, 1978. 268p.

BURKHOLDER, P.R. Antimicrobial Activity of some Marine Sponges. Reprinted from Nature. v.222, n..5197, p. 983-984, 1969.

GAMA, A .P.B. ; PEREIRA, R.C. Produtos não-poluentes contra a incrustação. Versão novembro de 1996. Disponível: http:// w.w.w. ciência.org.br.

HECHTEL, G. Zoogeography of Brazilian Marine Demospongiae. In: F. W. HARRISON ; R. R. COWDEN (eds). Aspects of Sponge Biology: p. 237-259, Academic Press, New York, 1976.

HOOPER, J.N. "Sponguide" Guide to sponge collection and identification. Version april 1998. Disponível: http://w.w.w.qmuseum.qld.gov.classification

JACCARD, P. Nouvelles recherches sur la distribution florale. Bulletin de la Societé Vandoise de Science Naturelle. n. 44, p. 223-270, 1908.

JOHNSON, M. F. Some Marine Sponges of Northeast Brazil. Arquivo de Ciências do Mar. v.11, n. 2, p. 103-116, 1971.

KELECOM, A. Marine organisms: an alternative source of potentially valuable natural products. Mem. Inst. Oswaldo Cruz,v. 86, n. 2, p. 99-106, 1991.

LAUBENFELS, M.W. de. Preliminary Discussion of the Sponge of Brazil. Contribuições Avulsas do Instituto Oceanográfico. São Paulo, n.1, p. 1-4, 1956.

LERNER, C.B.; SCHAPOVAL, E.; MOTHES, B.; HENRIQUES, A.; SCHWARTSMANN, G.; MANS, D.R.A. Anticancer Screening of SouthBrazilian Marine Sponges. Annals of Oncology, New York, v.9, Suplemento 2, p.35, july 1998.

LERNER, C.B.; MOTHES, B.; POSSUELO, L.G.; COSTA, J.C.; SCHAPOVAL, E.E.S., RECH, E.; FARIAS, F.M.; HENRIQUES, A.T.; MANS, D. Antimicrobial activity of sponges from southern Brazil, Atlantic coast. Memoirs of the Queensland Museum, Brisbane, v.44, p.306, 1999.

MOTHES, B. Esponjas da Plataforma Continental Norte e Nordeste do Brasil. (Porifera, Demospongiae). 233p. Tese (Doutorado em Zoologia). Universidade de São Paulo, São Paulo, 1996.

MURICY, G.; HAJDU, E.; ARAUJO, F. V.; HAGLER, A. N. Antimicrobial activity os southewestern Atlantic shallow-water marine sponges (Porifera). Scientia Marina. v. 57, n.4, p. 427-432, 1993.

ROHLF, F.J. ; FISHER, D.L. Test for hierarchical struture in random data sets Systematic Zoology, n. 17, p. 407-412, 1968.

SARÀ, M. ; VACELET, J. Ecologie des démosponges. In: P. GRASSÉ et al. (eds.). Spongiaires. Traité de Zoologie: anatomie, systématique, biologie: 3 (1): 462-576 Paris, Massom, 1973. 